\title{
Línea Espiral del Fémur
}

\author{
Spiral line of the Femur
}

Villarroel, M. ${ }^{1}$; Villagrán, F. ${ }^{2}$; Olave, E. ${ }^{2,3}$ \& Riveros, A.,4

\begin{abstract}
VILLARROEL, M.; VILLAGRÁN, F.; OLAVE, E. \& RIVEROS, A. Línea espiral del fémur. Int. J. Morphol., 38(1):193-198, 2020.
\end{abstract}
RESUMEN: La Linea aspera localizada en el margen posterior de la diáfisis del fémur, es considerada una referencia topográfica, imagenológica y antropológica. Hacia la epífisis proximal, esta línea se divide en varias líneas divergentes. De ellas, la rama lateral da inserción al músculo glúteo máximo (Tuberositas glutea), mientras que la rama media da inserción al músculo pectíneo (Linea pectinea). Ambas estructuras se encuentran en Terminologia Anatomica (TA), sin embargo, la rama medial conocida como línea espiral, donde se origina el musculo vasto medial (VM), no se encuentra en TA. Se realizó una revisión de la literatura del área morfológica con el objetivo de recopilar, analizar y verificar el uso del término línea espiral en nóminas, terminologías, textos de anatomía y publicaciones científicas. Se revisaron la Nomina Anatomica de Basilea, Paris Nomina Anatomica y Terminologia anatomica FCAT (2001) y FIPAT (2011). También fueron seleccionados 45 libros y 20 artículos en idiomas inglés y español, con el fin de analizar textos, tablas e imágenes del fémur y/o VM. Se confeccionaron tablas para resumir la información obtenida. Su término no es mencionado en nóminas ni terminologías, sin embargo en la columna de inglés de TA, se utiliza como sinónimo de Linea pectinea. En algunos textos anatómicos y artículos científicos se observa que el término línea espiral se utiliza en relación a la descripción del fémur y/o al origen del VM. El uso del término en textos de anatomía y publicaciones científicas, con fines descriptivos, antropológicos y clínicos, sumado al hecho que dentro de los principios de $T A$ el latín es el idioma oficial y se debe considerar un único nombre por término, con el fin de evitar confusiones, fundamentan la necesidad de incorporar a TA, el término línea espiral (Linea spiralis).

PALABRAS CLAVE: Anatomía; Fémur; Entesis; Línea espiral; Terminologia Anatomica.

\section{INTRODUCCIÓN}

En antropología física y forense se utiliza el concepto de entesis para identificar las áreas donde un tendón, ligamento o cápsula articular se inserta al hueso y actúan para transmitir una carga de tracción de los tejidos blandos al esqueleto (Apostolakos et al., 2014).

Anatómicamente se reconocen dos tipos de entesis: a) fibrosas, que corresponden a aquellas localizadas en diáfisis y en las que el músculo se adhiere directamente al hueso a través del periostio y b) fibrocartilaginosas, que se ubican en epífisis o procesos (Shaw \& Benjamin, 2007; Peña et al., 2012). Ambos tipos de entesis producen cambios en la remodelación ósea que se pueden evidenciar como tuberosidades, tubérculos, crestas, impresiones, surcos y líneas (Villotte et al., 2016). La causa de estas modificaciones entésicas dependen de diversos factores que incluyen el desgaste mecánico del hueso por estrés, condiciones patológicas, factores biológicos y estilo de vida (Slobodin et al., 2007; Villotte et al., 2010).
El fémur por su estratégica posición junto al cíngulo pélvico, presenta sitios de origen e inserción de músculos en su diáfisis y epífisis. La diáfisis del fémur presenta una Linea aspera (A02.5.04.013) (FCAT, 2001) de la cual surgen hacia su epífisis proximal dos líneas divergentes, la Tuberositas glutea (A02.5.04.017), que corresponde a la inserción del músculo glúteo máximo, y la Linea pectinea (A02.5.04.016) (Fig. 1A) que se genera por inserción del músculo pectíneo. Por otra parte, el labio medial de la Linea aspera se continúa hacia proximal como línea espiral, entesis que da origen del músculo vasto medial (Llusá et al., 2004) (Fig. 1B).

La línea espiral no se encuentra incorporada en Terminologia Anatomica (TA), según consta en el texto oficial que es divulgada por el Programa Federativo Internacional de Terminología Anatómica (FIPAT) y aprobado por la Federación Internacional de Asociaciones de Anatomía (IFAA) y que en el año 2001 se publicó traducido al español por la Sociedad Española de Anatomía (Tabla I).

\footnotetext{
${ }^{1}$ Laboratorio de Morfología, Departamento Disciplinario de Biología, Universidad de Playa Ancha, Valparaíso, Chile.

${ }^{2}$ Programa de Doctorado en Ciencias Morfológicas, Universidad de La Frontera, Chile.

${ }^{3}$ Facultad de Medicina, Universidad de La Frontera, Temuco, Chile.

${ }^{4}$ Departamento de Ciencias Morfológicas, Facultad de Medicina y Ciencia, Universidad San Sebastián, Lientur 1457, Concepción 4080871, Chile.
} 
Tabla I. Estructuras asociadas al línea espiral en Terminologia Anatomica, su traducción al inglés y español.

\begin{tabular}{lccc}
\hline Número identificador & Términos en Latín & Términos en Inglés & Términos en Español \\
\hline A02.5.04.012 & Corpus femoris & Shaft of femur; Body of femur & Cuerpo \\
A 02.5 .04 .013 & Linea aspera & Linea aspera & Línea áspera \\
A 02.5 .04 .014 & Labium laterale & Lateral lip & Labio lateral \\
A 2.5 .04 .015 & Labium mediale & Medial lip & Labio medial \\
A 2.5 .04 .016 & Linea pectinea & Pectineal line; Spiral line & Línea pectínea \\
A02.5.04.017 & Tuberositas glutea & Gluteal tuberosity & Tuberosidad glútea \\
\hline
\end{tabular}
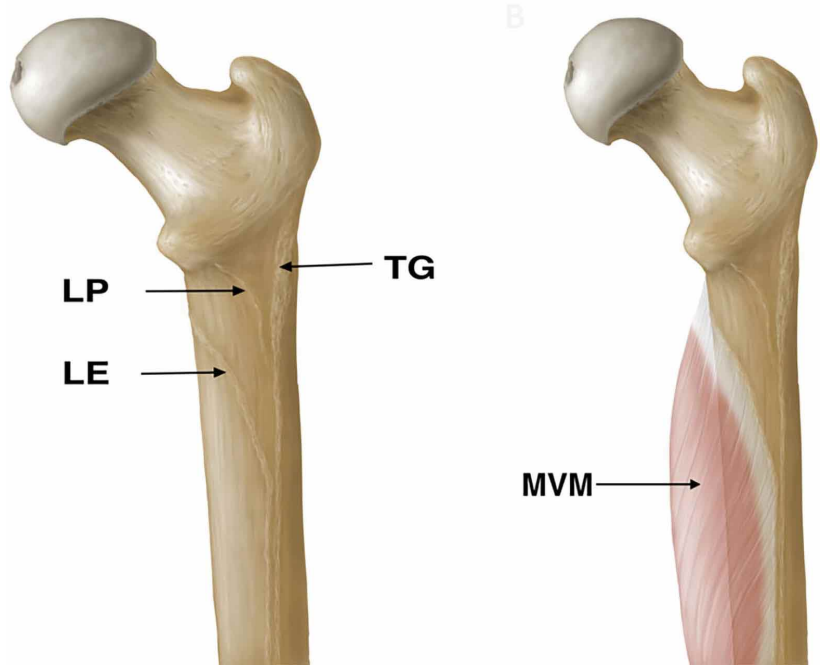

Fig. 1. A Fémur derecho humano, vista posterior; TG: Tuberositas glutea, LP: Linea pectinea y LE: Línea espiral. B. Fémur derecho humano y el origen del músculo vasto medial del músculo cuádriceps femoral, vista posterior; MVM: músculo vasto medial.
Con el objetivo de recopilar, analizar y verificar el uso del término línea espiral en la literatura del área morfológica, se realizó una revisión de nóminas, terminologías, publicaciones científicas y textos de anatomía empleados a nivel universitario.

\section{MATERIAL Y MÉTODO}

Se realizó una revisión de la literatura, la cual se separó en tres etapas. En la primera de ellas, se analizaron: a) Nomina Anatomica de Basilea (BNA) (His, 1895), b) Paris Nomina Anatomica (PNA) (IANC, 1955), c) Terminologia Anatomica, del año 2001, publicada por la IFAA y la Sociedad Anatómica Española (FCAT, 2001) y d) Terminologia Anatomica (FIPAT, 2011). En cada una de ellas se estudiaron los términos vinculados con la morfología del fémur y en particular con la línea espiral: a) Linea aspera, b) Tuberositas glutea y c) Linea pectinea.

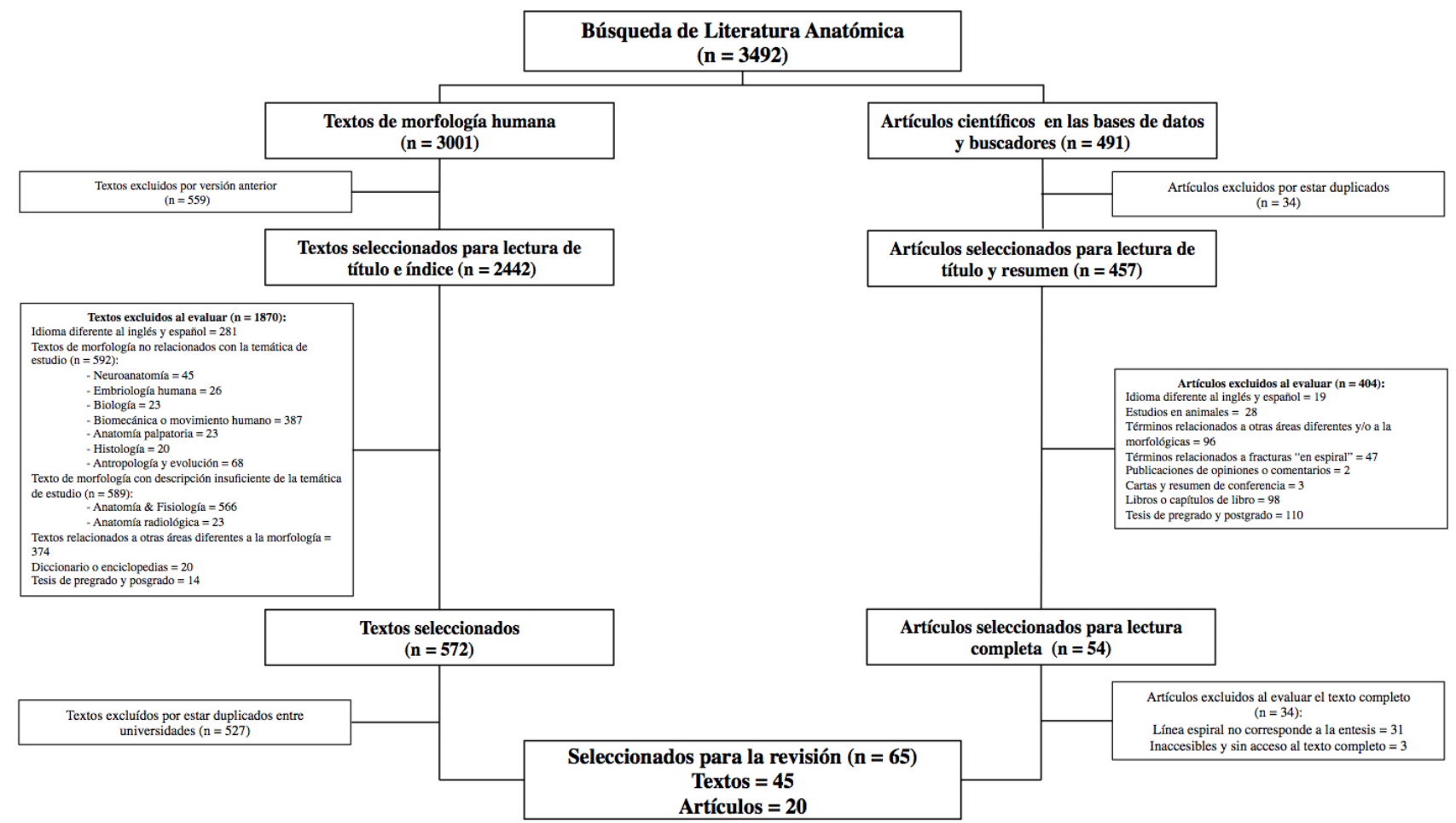

Fig. 2. Flujograma del proceso de búsqueda bibliográfica y selección de textos de morfología humana y artículos científicos. 
En una segunda etapa se revisó la literatura científica, mediante un estudio descriptivo de 45 libros de morfología humana (textos, tratados, compendios y atlas), editados en los últimos 50 años, en idiomas español e inglés. Dicha selección consideró la edición más actualizada de los textos que se encuentran en los catálogos del Sistema de Bibliotecas del Consejo de Rectores de Universidades Chilenas. En cada uno de ellos se verificó el uso de los términos anteriormente mencionados, analizando el texto, las imágenes y las tablas de los capítulos de osteología general, miología y/o anatomía topográfica del miembro inferior. Con lo anterior se determinó la frecuencia de utilización del término línea espiral o spiral line.

Finalmente, en la tercera etapa consistió en una búsqueda de artículos científicos, los que se obtuvieron en las bases de datos Web of Science, PubMed, Scopus, SciELO y LILACS y en el buscador Google Académico (Fig. 2). Se utilizaron como palabras clave los términos en español e inglés, línea espiral (spiral line), "fémur" (femur), "músculo vasto medial" (vastus medialis muscle), combinados con el término boleano AND. La selección de los artículos se realizó de acuerdo criterios de exclusión que están detallados en la Figura 2.

Toda la información recopilada fue tabulada en el Software Microsoft Excel®365, realizando el análisis de utilización de los términos en frecuencia y porcentaje.

\section{RESULTADOS}

$\mathrm{Al}$ analizar y comparar las nóminas y terminologías, en la primera de ellas, BNA (1895), no se incluye la línea espiral en la descripción de las formaciones óseas del fémur. Esto se reitera en la PNA (1955) y en TA $(2001,2011)$, ambas publicadas por la IFAA.
Puntualmente en TA del 2001 se enlistan los términos en latín, inglés y español, aquí destaca que en la columna en inglés, la línea espiral es utilizada como sinónimo de Linea pectinea (Tabla I), situación similar ocurre en la $T A$ del 2011.

De los 45 textos de anatomía revisados, el 42,2 \% de ellos utiliza en su descripción el término línea espiral. Al realizar un desglose de esta información, un 47,4 \% lo menciona en la parte escrita y en tablas y/o imágenes, un 36,8 \% lo menciona solo en texto o tablas, y un $15,8 \%$ solamente en el rotulado de la imagen del fémur. En relación a los 20 artículos científicos seleccionados, el $80 \%$ utiliza el término línea espiral en el texto, tablas e imágenes, un $10 \%$ solo lo menciona en imágenes y el mismo porcentaje, en tablas o textos.

Los diferentes términos utilizados por los textos de anatomía para referirse a la línea espiral se encuentran resumidos en la Tabla II.

\section{DISCUSIÓN}

En la anatomía humana, Terminologia Anatomica sirve como una herramienta única de comunicación, tanto en disciplinas médicas, como en otros campos científicos. Dicha terminología, establece un orden en sus términos y evita confusiones generadas por la multiplicidad de ellos para señalar a una misma estructura. Producto de lo anterior, la creación de un único cuerpo lingüístico para esta disciplina ha sido el principal desafío de los morfólogos. Reflejo de ello, fue que en 1895 la Anatomische Gesellschaft se reunió en Basilea, Suiza, logrando generar la Basle Nomina Anatomica (BNA) (His), la cual fue escrita en latín (Cruz et al., 2010). El uso de este idioma como base se mantuvo tanto en la Nomina Anatomica aprobada en Paris en 1955 (PNA), como en cada una de las seis ediciones que le siguieron.

Tabla II. Términos utilizados por los textos de anatomía para referirse a la línea espiral.

\begin{tabular}{lll}
\hline Término & Término utilizado en la literatura científica & \multicolumn{1}{c}{ Autores } \\
\hline Línea espiral & Cresta del músculo vasto medial & Testut \& Latarjet, 1972; García-Porrero \& Hurlé, \\
& 2005, Rouvière \& Delmas, 2005. \\
Labio medial de la Linea aspera & Chatain \& Bustamante, 1986; Kahle et al., 1995, \\
& Pansky, 1998; Dufour, 2003; *Schünke et al., 2005. \\
& * Sinea pectinea & $*$ Standring, 2008, *Moses et al., 2013.
\end{tabular}


Actualmente TA, aprobada por la IFAA en 1989 presenta una columna en latín y otra con su traducción al inglés. En el 2001, la Sociedad Anatómica Española le incorporó una tercera columna con su traducción al español (Riveros et al., 2019).

Esta TA sigue los principios básicos establecidos por la IFAA consisten en: a) las estructuras morfológicas se denominen solo con un término, b) que los nombres de las estructuras tengan un valor informativo y c) se suprimen el uso de epónimos. En este sentido, es importante considerar que al suprimir los homónimos se evitan confusiones y se tiende a que las estructuras de las mismas regiones anatómicas tengan nombres armonizados (Vásquez \& del Sol, 2015). Sin embargo, a pesar de lo trabajado, aún quedan algunos puntos débiles que merecen ser discutidos, persistiendo algunos errores, discrepancias, multiplicación de términos, sinónimos, términos idénticos para diferentes estructuras, términos demasiado extensos y términos ausentes (Kachlik et al., 2015; Villarroel et al., 2016). Dicha labor de revisión ha sido abordada en los Simposios Iberolatinoamericanos de Anatomia, Histologia y Embryologia (SILAT), en los cuales se han analizado los términos incluidos en Terminologia Anatomica, Histologica y Embryologica, proponiendo correcciones en los caso que amerite (Cruz et al.; Losardo et al., 2015).

Uno de los términos ausentes en $T A$ en su idioma oficial es la línea espiral, estructura anatómica que aparece en el 42,2\% de nuestra muestra en los textos de anatomía. Si bien el porcentaje no alcanza el $50 \%$ de la muestra, si ha sido incluida por autores influyentes como Testut \& Latarjet o Rouvière \& Delmas. En ambos casos se describe una trifurcación proximal de la Linea aspera, denominando la formación medial de esta trifurcación como cresta del músculo vasto medial (Tabla II). Es importante destacar, que prácticamente en todas las imágenes de la epífisis proximal del fémur revisadas en la literatura, aparece representada la entesis del músculo vasto medial, indicando o no un término asociado (Feneis \& Dauber, 2000).

El caso del texto Gray's Anatomy: The Anatomical Basis of the Clinical Practice, 40st edición y editado por Susan Standring es bastante particular; su descripción refleja la misma confusión que se evidencia en la TA (Tabla II), en la cual la línea espiral es mencionada como sinónimo de Linea pectinea. Sin embargo, al revisar las imágenes de este texto, se describe un labio medial de la Linea aspera, como aquella formación que se continúa hacia proximal con la Linea intertrocanterea (Standring).

En cuanto a las descripciones morfológicas en artículos científicos, el $80 \%$ de los artículos revisados incluye en su descripción a la línea espiral. Varios de ellos, conside- rando la ausencia de un término oficial, se basaron en la descripción de Moore et al., (2013) por ejemplo Polguj et al., (2013) describe la Linea aspera dividiéndose proximalmente en dos labios, el labio lateral se vuelve continuo con la Tuberositas glutea, el labio medial se divide en dos líneas separadas: la línea espiral y la Linea pectinea. Esta descripción fue reafirmada por Kassem et al., (2016) quienes al describir la Linea aspera, diferenciaron claramente la línea espiral de la Linea pectinea. Al respecto Raj et al., (2018) y Suthar et al., (2015), señalan que la línea espiral presenta una continuidad con la línea intertrocantérica.

Lo descrito anteriormente está en sintonía con los artículos que describen el origen del músculo vasto medial, en donde detallan que una fracción de su origen que se ubica en línea espiral (Vergara \& Roman, 2011; Rajput et al., 2017). Al respecto, el mismo Polguj diferencia claramente la línea espiral de la Linea pectinea, señalando que mientras la primera proporciona el origen del músculo vasto medial, la segunda marca la inserción del músculo pectíneo, la cual se sitúa lateral y superior a la línea espiral.

$\mathrm{Al}$ igual que otras formaciones anatómicas del fémur, la línea espiral también es utilizada como punto de referencia para determinar la ubicación de los forámenes nutricios en el fémur (Mysorekar, 1967; Sharma et al., 2015). Lo anterior toma importancia, ya que se han observado forámenes nutricios en la misma línea espiral, por lo que la precisión de la ubicación de éstos puede ser de utilidad en los procedimientos quirúrgicos, ya sea para injertos óseos, como también en transplantes de hueso (Agrawal et al., 2016; Vijayalakshmi et al., 2016).

Por otra parte, en manuscritos de carácter antropológico forense, esta entesis, que también denominan línea espiral ha sido analizada junto a otras formaciones óseas como parámetro válido para estimar la edad de muerte en restos esqueléticos humanos de acuerdo a sus modificaciones y grados de marcaje (Listi, 2016). En este mismo sentido, Haeusler et al., (2004) mencionaron a la línea espiral como marca muscular presente en fémures de homínidos como en el caso del Homo habilis.

En el área de traumatología, la línea espiral también toma importancia principalmente al momento de realizar diagnósticos diferenciales de patologías óseas que afectan la epífisis proximal del fémur. Lo anterior queda en evidencia, ya que al analizar los signos radiológicos que permiten confirmar la presencia de zonas líticas que se desarrollan en la enfermedad de Paget u osteítis deformante, es necesario conocer la normalidad o variaciones que presenta la epífisis proximal del fémur, permitiendo generar un diagnóstico diferencial eficiente de la patología de cadera (Quintana et al., 2014). 
Basado en la premisa de que un término anatómico debe ser consecuente respecto a su precisión y claridad, no creando contradicciones (Villarroel et al.) el término línea espiral es utilizado en la literatura científica pero su descripción es discordante. Detalles como el uso de otros términos y homologar la línea espiral con la Linea pectinea son muestra de ello. El papel del latín en TA como base para la comprensión sigue siendo insustituible, y si bien se han reducido sucesivamente varios sinónimos creando y adoptando una lista de términos única, uniforme y coherente, pero desafortunadamente en este caso no existe consenso en el uso de la línea espiral en el inglés (Kachlik et al., 2008).

Kachlik et al., (2015) clasificaron en siete grupos los términos incorrectos o inexactos presentados en TA. Uno de ellos se refiere al uso de sinónimos, generalmente se presentan dos términos, excepcionalmente tres. Lo anterior se explica por una posición dominante del inglés. Este factor puede mantener las confusiones y dificultar la búsqueda, especialmente cuando se utilizan herramientas electrónicas. En este sentido, Kachlik et al., (2017) presentaron una propuesta de 69 términos en latín y sus equivalentes en inglés concernientes a estructuras de miembro inferior. Entre sus propuestas de incorporación a la nueva versión de $T A$, está presente la línea espiral del fémur (Linea spiralis femoris) en inglés y en latín.

En conclusión, el uso del término línea espiral tanto en textos de anatomía, como en publicaciones científicas, sumado a su utilidad para determinar el origen del músculo vasto medial, le confiere a esta entesis un valor anatómico, antropológico y clínico. Por lo tanto, se fundamenta considerar la incorporación del término Linea spiralis a Terminologia Anatomica.

\section{AGRADECIMIENTOS}

Agradecemos al ilustrador Alfredo Torres Parra por su contribución gráfica a esta comunicación científica.

VILLARROEL, M.; VILLAGRÁN, F.; OLAVE, E. \& RIVEROS, A. Spiral line of the fémur. Int. J. Morphol., 38(1):193198, 2020 .

SUMMARY: The Linea aspera located on the posterior margin of the femur diaphysis is considered a tomographic, imaging and anthropological reference. To the proximal epiphysis, this line is divided in several divergent lines. Of these, the lateral branch gives insertion to the gluteus maximus muscle (Tuberositas glutea), while the middle branch gives insertion to the pectineal muscle (pectinea line). Both structures are found in Terminologia
Anatomica (TA), however the medial branch known as the Spiral Line, where the vastus medial muscle (VM) originates, is not found in $T A$. We aimed to collect, analyze and verify the level of use of the term Spiral line in terminologies, anatomy texts and scientific publications. Nomina Anatomica from Basilea (BNA), Paris Nomina Anatomica (PNA) y Terminologia anatomica FCAT (2001) and FIPAT (2011) were reviewed. In addition, 45 books and 20 articles in English and Spanish were also selected, in order to analyze text, tables and images of the femur and/or VM. Tables were made to summarize the information obtained. The term is not mentioned in payrolls or terminologies, however in the English column of $T A$, this term is used as a synonym for Linea pectinea. In some anatomical texts and scientific articles it is observed that the term spiral line is used in relation to the description of the femur and / or the origin of the vast medial. The use of the term in anatomy texts and scientific publications, for descriptive, anthropological and clinical purposes, added to the fact that within the principles of Anatomical Terminology Latin is the official language and should be considered a single name per term, in order to avoid confusion, they base the need to incorporate the term spiral line (Linea spiralis) into $T A$.

KEY WORDS: Anatomy; Femur; Enthesis; Spiral line; Terminologia Anatomica.

\section{REFERENCIAS BIBLIOGRÁFICAS}

Agrawal, N.; Tiwari, A. \& Naik, D. C. An analytical study of number, position, size and direction of nutrient foramina of femur. Int. J. Med. Sci. Public. Health., 5(3):489-92, 2016.

Apostolakos, J.; Durant, T.; Dwyer, C.; Russell, R.; Weinreb, J.; Alaee, F.; Beitzel, K.; McCarthy, M.; Cote, M. \& Mazzocca, A. The enthesis: a review of the tendon-to-bone insertion. Muscles Ligaments Tendons J., 4(3):333-42, 2014.

Comité Federativo sobre Terminología Anatómica (FCAT). Terminologia anatomica. Buenos Aires, Médica Panamericana, 2001.

Cruz, R.; Rodríguez, A.; Prates, J.; Losardo, R. \& Prates, N. Ibero-LatinAmerican Symposium of Morphological Terminology. General characteristics. Int. J. Morphol., 28(2):637-42, 2010.

Chatain, I. \& Bustamante, J. Anatomía macroscópica funcional y clínica. Ciudad de México, Addison Wesley Iberoamericana, 1986.

Federative International Programme on Anatomical Terminologies (FIPAT). Terminologia Anatomica. 2st ed. New York, Thieme, 2011.

Feneis, H. \& Dauber, W. Pocket Atlas of Human Anatomy. Based on the International Nomenclature. 4st ed. Stuttgart, Thieme Verlag, 2000.

García-Porrero, J. \& Hurlé, J. Anatomía Humana. Madrid, McGrawHill, 2005.

Haeusler, M. \& Mchenry, H. Body Proportions of Homo habilis Reviewed. Journal of human evolution. J. Hum. Evol., 46(4):33-65, 2004.

His, W. Nomina Anatomica. Leipzig, Verlag von Veit \& Comp., 1895.

International Anatomical Nomenclature Committee. Nomina Anatomica. 2a ed. Aprobada en el 60 y 7 o International Congresses of Anatomists, París (1955) y New York (1960). Amsterdam, Excerpta Medica Foundation. 1961.

Kachlik, D.; Baca, V.; Bozdechova, I.; Cech, P. \& Musil, V. Anatomical terminology and nomenclature: past, present and highlights. Surg. Radiol. Anat., 30(6):459-66, 2008.

Kachlik, D.; Musil, V. \& Baca, V. Terminologia Anatomica after 17 years: Inconsistencies, mistakes and new proposals. Ann. Anat., 201:8-16, 2015. 
Kachlik, D.; Musil, V. \& Baca, V. Contribution to the anatomical nomenclature concerning upper limb anatomy. Surg. Radiol. Anat., 39(4):405-17, 2017.

Kahle, W.; Leonhardt, H. \& Platzer, W. Atlas de Anatomía para estudiantes y médicos. 5ta ed. Barcelona, Omega, 1995.

Kassem, K.; Yamamoto, N.; Hayashi, K.; Takeuchi, A; Miwa, S.; Fawaz, A.; Kajino, Y. \& Tsuchiya, H. The linea aspera as a guide for femoral rotation after tumor resection: is it directly posterior? A technical note. J. Orthop. Traumatol., 17(3):255-9, 2016.

Lippert, H. Anatomía. Vol. I. 4ta ed. Madrid, Marbán, 2002.

Listi, G. The Use of Entheseal Changes in the Femur and Os Coxa for Age Assessment. J. Foren. Scien., 61(1):12-18, 2016.

Llusá, M.; Merí, A. \& Ruano Gil, D. Manual y atlas fotográfico de anatomía del aparato locomotor. Buenos Aires, Médica Panamericana, 2004

Losardo, R.; Prates, N.; Arteaga-Martínez, M.; Cabral, R. H. \& GarcíaPeláez, M. Terminología Morfológica Internacional: Algo Más que Anatomía, Histología y Embriología. Int. J. Morphol., 33(1):400-7, 2015.

Moore, K.; Dailey, A. \& Agur, A. Anatomía con orientación clínica. Wolters Kluver/Lippincott. Buenos Aires, Williams \& Wilkins, 2013.

Moses, K.; Banks, J.; Nava, P \& Petersen, D. Atlas clínical Gross Anatomy. Philadelphia, Elsevier, 2013.

Mysorekar, V. R. Diaphysial nutrient foramina in human long bones. $J$. Anat., 101(Pt 4):813-822, 1967.

Pansky, B. Anatomía Humana. México, McGraw-Hill Interamericana, 1998.

Peña, P.; Páramo, E.; Varela, P.; Valle, R. \& Patiño, J. Entesis, entesopatía y espondiloartritis. Rev. Colom. Reumatol., 19(1):19-26, 2012.

Polguj, M.; Bliz'niewska, K.; Je drzejewski, K.; Majos, A. \& Topol, M. Morphological study of linea aspera variations-proposal of classification and sexual dimorphism. Folia. Morphol., 72(1):72-7, 2013.

Quintana, I.; Mellado, J.; Salceda, J.; Yanguas, N.; Martín, J.; , I. Sanmartin, I. \& Tudela, E. Anatomía y variantes normales del fémur proximal: hallazgos en TCMD. Oviedo, 32 Congreso de la SERAM, 2014. Disponible en: https://pdfs.semanticscholar.org/0667/ b1d28ab2f0db4750d0e2f09083cd840ebe01.pdf

Raj, V.; Arokiaraj, M. \& Xavier, A. Functional and radiological outcome of intertrochanteric fracture treated with proximal femoral nail. Int. J. Orthop. Sci., 4(1):670-3, 2018.

Rajput, H.; Rajani, S. \& Vaniya, V. Variation in morphometry of vastus medialis muscle. J. Clin. Diagn. Res., 11(9):AC01-AC04, 2017.

Riveros, A.; Olave, E.; Nicholson, C.; Schorwer, K. \& Torres, H. Communicating branches between the median and ulnar nerves proposal for inclusion in Terminologia Anatomica. Int. J. Morphol., 37(3):1192-96, 2019.

Rouvière, H. \& Delmas, A. Anatomía Humana Descriptiva, Topográfica y Funcional. Vol. III. 11ta ed. Barcelona, Masson, 2005.

Schünke, M.; Schulte, M. \& Schumacher, U. Prometheus: texto y atlas de anatomía. Buenos Aires, Panamericana, 2005.

Sharma, M.; Prashar, R.; Sharma, T.; Wadhwa, A. \& Kaur, J. Morphological variations of nutrient foramina in lower limb long bones. Int. J. Med. Dent. Sci., 4(2):802-8, 2015.

Shaw, H. \& Benjamin, M. Structure-function relationships of entheses in relation to mechanical load and exercise. Scan. J. Med. Sci. Sports., 17(4):303-15, 2007.

Sinelnikov, R. Atlas of human anatomy. Moscow, Mir Publisher, 1986.

Slobodin, G.; Rozenbaum, M.; Boulman, N. \& Rosner, I. Varied presentations of enthesopathy. Semin. Arthritis. Rheum., 37:119-26, 2007

Standring, S. Gray's Anatomy: The Anatomical Basis of the Clinical Practice. 40st ed. Edinburg, Elsevier Churchill Livingstone, 2008.

Suthar, P.; Patel, C.; Gamit, M.; Dave, D.; Wadhwani, C. \& Suthar, P. Orthopaedic aspect of anatomy and radiology of proximal femur. Int. J. Res. Med. Sci., 3(8):1820-24, 2015.
Testut, L. \& Latarjet, A. Tratado de Anatomía Humana. Vol I. 9ta ed. Barcelona, Salvat, 1972.

Vásquez, B. \& del Sol, M. Terminologia Anatomica y Terminologia Histologica: Un Lugar de Encuentro entre los Morfólogos. Int. J. Morphol., 33(4):1585-90, 2015.

Vergara, E. \& Román, M. Descripción anatómica del músculo vasto medial: ¿Existe realmente el músculo vasto medial oblicuo?. Salud Uninorte, 27(1):73-84, 2011

Vijayalakshmi, S.; Kalthur, S. \& D' Souza, A. A study of diaphyseal nutrient foramina in human lower limb long bones and its clinical importance. Int. J. Curr. Res. Med. Sci., 2(7):1-12, 2016.

Villarroel, M.; Acuña, C.; Olguín, C. \& Velásquez, F. Tubérculo deltoideo: Discrepancias entre la terminología anatómica y bibliografía anatómica. Int. J. Morphol., 34(4):1318-21, 2016.

Villotte, S.; Castex, D.; Couallier, V.; Dutou, O.; Knüsel, C. \& HenryGambier, D. Enthesopathies as occupational stress markers: evidence from the upper limb. Am. J. Phys. Anthropol., 142(2):224-34, 2010.

Villotte, S.; Assis, S.; Alves, F.; Henderson, C.; Mariotti, V.; Milella, M.; Pany-Kucera, D.; Speith, N.; Wilczak, C. \& Jurmain, R. In search of consensus: Terminology for entheseal changes (EC). Int. J. Paleopathol., 13:49-55, 2016.

Dirección para correspondencia:

Dr. Mauricio Villarroel Guerra

Departamento Disciplinario de Biología

Facultad de Ciencias Naturales y Exactas

Universidad de Playa Ancha

Casilla 34-V

Valparaíso

CHILE

Email: mvillarr@upla.cl

Recibido : 06-09-2019

Aceptado: 11-10-2019 